DOE/ER/40762-259

UMD-PP\#02-059

\title{
Nucleon hologram with exclusive leptoproduction
}

\author{
A.V. Belitsky ${ }^{a}$, D. Müller ${ }^{b}$ \\ ${ }^{a}$ Department of Physics \\ University of Maryland at College Park \\ College Park, MD 20742-4111, USA \\ ${ }^{a}$ Fachbereich Physik \\ Universität Wuppertal \\ D-42097 Wuppertal, Germany
}

\begin{abstract}
Hard exclusive leptoproductions of real photons, lepton pairs and mesons are the most promising tools to unravel the three-dimensional picture of the nucleon, which cannot be deduced from conventional inclusive processes like deeply inelastic scattering.
\end{abstract}

Brief summary of talks given at

17th Summer School HUGS@CEBAF (Jefferson Lab, June 2002)

Workshop "Exclusive processes at high momentum transfer" (Jefferson Lab, May 2002)

Workshop "QCD structure of the nucleon" (Ferrara, April 2002)

Workshop "Testing QCD through spin observables" (Charlottesville, April 2002)

Conference "Baryons 2002" (Jefferson Lab, March 2002)

"Electron-Ion Collider Workshop" (Brookhaven Lab, February 2002) 


\title{
Nucleon hologram with exclusive leptoproduction
}

\author{
A.V. Belitsky ${ }^{\mathrm{a}}$, D. Müller ${ }^{\mathrm{b}}$ \\ a Department of Physics, University of Maryland, College Park, MD 20742-4111, USA \\ b Fachbereich Physik, Universität Wuppertal, D-42097 Wuppertal, Germany
}

Hard exclusive leptoproductions of real photons, lepton pairs and mesons are the most promising tools to unravel the three-dimensional picture of the nucleon, which cannot be deduced from conventional inclusive processes like deeply inelastic scattering.

\section{From macro to micro}

Why do we see the world around us the way it is? Human eyes can detect electromagnetic waves in a very narrow range of wavelength, $\lambda_{\gamma} \sim 0.4-0.7 \mu \mathrm{m}$, which we call visible light. The light from a source, say the sun, is reflected from the surface of macro-objects and is absorbed by the eye's retina which transforms it into a neural signal going to the brain which forms the picture. The same principle is used in radars which detect reflected electromagnetic waves of a meter wavelength. The only requirement to "see" an object is that the length of resolving waves must be comparable to or smaller than its size. The same conditions have to be obeyed in case one wants to study the microworld, e.g., the structure of macromolecules (DNA, RNA) or assemblies (viruses, ribosomes). Obviously, when one puts a chunk of material in front of a source of visible light, see Fig. 1, the object merely leaves a shadow on a screen behind it and one does not see its elementary building blocks, i.e., atoms. Obviously, visible light is not capable to resolve the internal lattice structure of a crystal since the size of an individual atom, say hydrogen, is of order $r_{\text {atom }} \sim\left(\alpha_{\text {em }} m_{e}\right)^{-1} \sim(10 \mathrm{KeV})^{-1}$ and the light does not diffract from it. Therefore, to "see" atoms in crystals one has to have photons with the wavelength $\lambda_{\gamma} \leq r_{\text {atom}}$, or equivalently, of the energy $E_{\gamma} \geq r_{\text {atom. }}^{-1}$. To do this kind of "nano-photography" one needs a beam of X-rays which after passing through the crystal creates fringes on a photo-plate, see Fig. 1. Does one get a three-dimensional picture from such a measurement? Unfortunately, no. In order to reconstruct atomic positions in the crystal's lattice one has to perform an inverse Fourier transform. This requires knowledge of both the magnitude and the phase of diffracted waves. However, what is measured experimentally is essentially a count of number of X-ray photons in each spot of the photo-plate. The number of photons gives the intensity, which is the square of the amplitude of diffracted waves. There is no practical way of measuring the relative phase angles for different diffracted spots experimentally. Therefore, one cannot unambiguously reconstruct the crystal's lattice. This is termed as "The Phase Problem". None of techniques called to tackle the problem provides a parameter-free answer. 

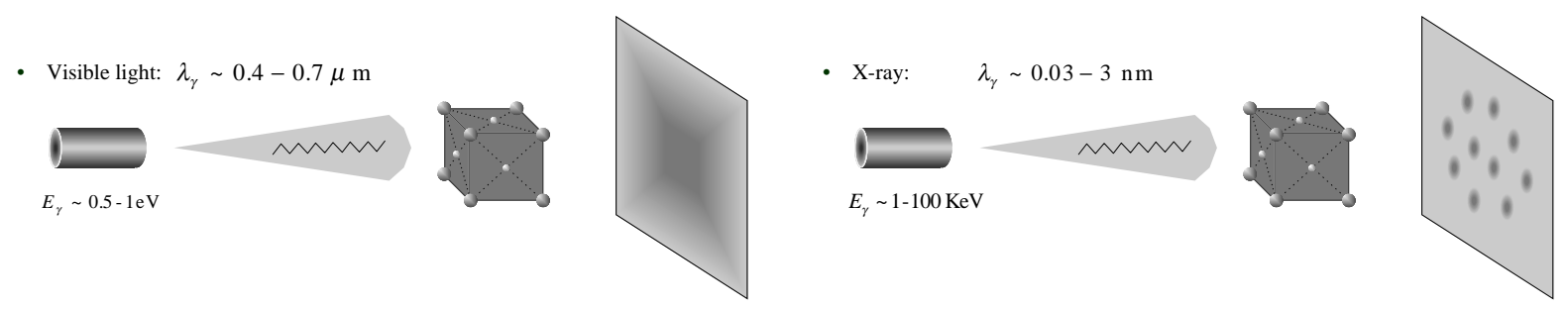

Figure 1. Left: A beam of visible light does not resolve the crystal's structure. Right: An X-ray beam does and creates a diffraction pattern on the photo-plate.

When we study hadronic matter at the fundamental level we attempt to perform the "femto-photography" of the interior constituents (quarks and gluons) of strongly interacting "elementary" particles such as the nucleon. Quantum $\chi \rho \omega \mu \alpha$ dynamics, the theory of strong interaction, is not handy at present to solve the quark bound state problem. Therefore, phenomenological approaches, based on accurate analyses of high-energy scattering experimental data and making use of rigorous perturbative QCD predictions, are indispensable for a meticulous understanding of the nucleon's structure. As we discuss below most of high-energy processes resolving the nucleon content, such as described in terms of form factors and inclusive parton densities, suffer from the same "Phase Problem" and therefore they lack the opportunity to visualize its three-dimensional structure. A panacea is found in newborn generalized parton distributions [1], which are measurable in exclusive leptoproduction experiments.

\section{Form factors}

Nucleon form factors are measured in the elastic process $\ell N \rightarrow \ell^{\prime} N^{\prime}$. Its amplitude is given by the lepton current $L_{\mu}(\Delta) \equiv \bar{u}_{\ell}(k-\Delta) \gamma_{\mu} u_{\ell}(k)$ interacting via photon exchange with the nucleon matrix element of the quark electromagnetic current $j_{\mu}(x)=\sum_{q} e_{q} \bar{q}(x) \gamma_{\mu} q(x)$ :

$\mathcal{A}_{N N^{\prime}}=\frac{1}{\Delta^{2}} L_{\mu}(\Delta)\left\langle p_{2}\left|j_{\mu}(0)\right| p_{1}\right\rangle \equiv \frac{1}{\Delta^{2}} L_{\mu}(\Delta)\left\{h_{\mu} F_{1}\left(\Delta^{2}\right)+e_{\mu} F_{2}\left(\Delta^{2}\right)\right\}$.

Here the matrix element of the quark current is decomposed in terms of Dirac and Pauli form factors $\left(\Delta \equiv p_{2}-p_{1}\right)$, accompanied by the Dirac bilinears $h_{\mu} \equiv \bar{u}_{N}\left(p_{2}\right) \gamma_{\mu} u_{N}\left(p_{1}\right)$ and $e_{\mu} \equiv \bar{u}_{N}\left(p_{2}\right) i \sigma_{\mu \nu} \Delta_{\nu} u_{N}\left(p_{1}\right) /\left(2 M_{N}\right)$. In the Breit frame $\vec{p}_{2}=-\vec{p}_{1}=\vec{\Delta} / 2$ there is no energy exchange $E_{1}=E_{2}=E$ and thus relativistic effects are absent. The momentum transfer is three-dimensional $\Delta^{2}=-\vec{\Delta}^{2}$, so that

$\left\langle p_{2}\left|j_{0}(0)\right| p_{1}\right\rangle=\tilde{\varphi}_{2}^{*} \tilde{\varphi}_{1} G_{E}\left(-\vec{\Delta}^{2}\right), \quad\left\langle p_{2}|\vec{j}(0)| p_{1}\right\rangle=-\frac{i}{2 M_{N}} \tilde{\varphi}_{2}^{*}[\vec{\Delta} \times \vec{\sigma}] \tilde{\varphi}_{1} G_{M}\left(-\vec{\Delta}^{2}\right)$,

are expressed in terms of Sachs electric $G_{E}\left(\Delta^{2}\right) \equiv F_{1}\left(\Delta^{2}\right)+\Delta^{2} /\left(4 M_{N}^{2}\right) F_{2}\left(\Delta^{2}\right)$ and magnetic $G_{M}\left(\Delta^{2}\right) \equiv F_{1}\left(\Delta^{2}\right)+F_{2}\left(\Delta^{2}\right)$ form factors. Introducing the charge $q \equiv \frac{1}{V} \int d^{3} \vec{x} j_{0}(\vec{x})$ and magnetic moment $\vec{\mu} \equiv \frac{1}{V} \int d^{3} \vec{x}[\vec{x} \times \vec{j}](x)$ operators, one finds the normalization

$\langle p|q| p\rangle=\tilde{\varphi}_{2}^{*} \tilde{\varphi}_{1} G_{E}(0), \quad\langle p|\vec{\mu}| p\rangle=\frac{\tilde{\varphi}_{2}^{*} \vec{\sigma} \tilde{\varphi}_{1}}{2 M_{N}} G_{M}(0)$. 

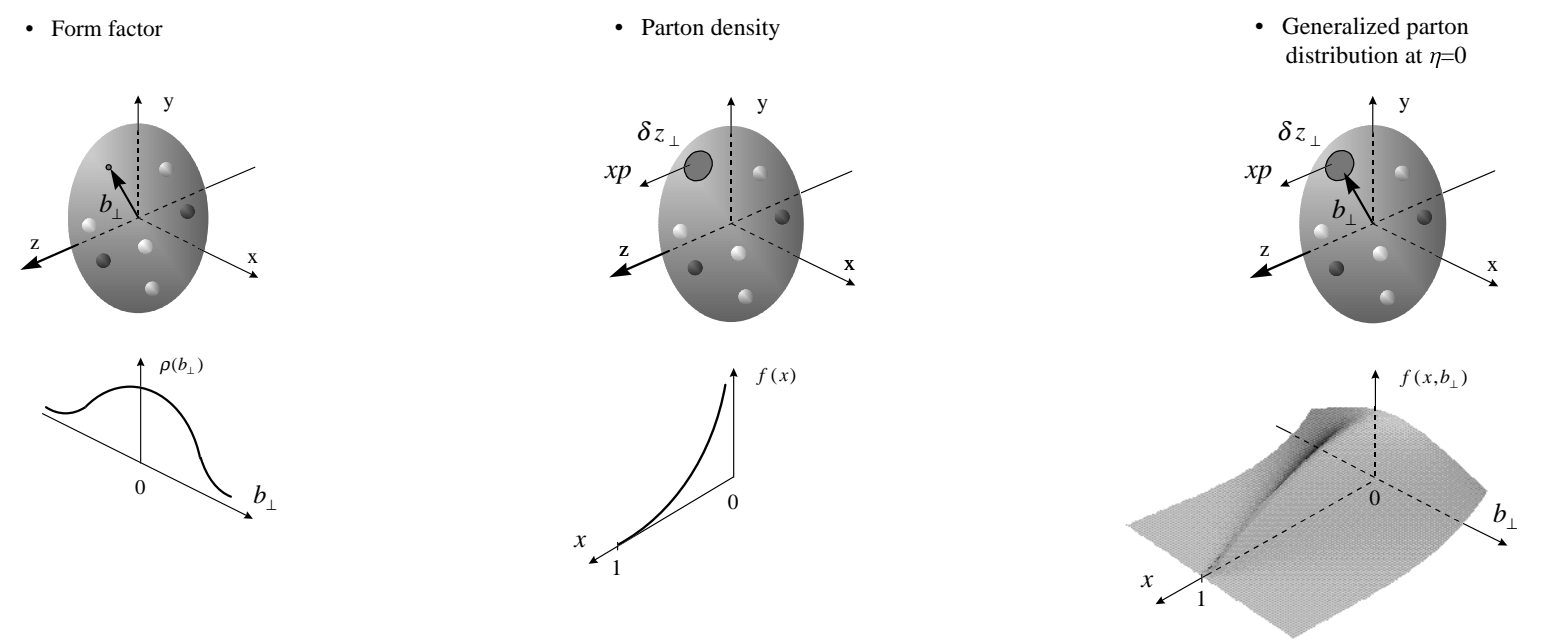

Figure 2. Probabilistic interpretation of form factors, parton densities and generalized parton distributions at $\eta=0$ in the infinite momentum frame $p_{z} \rightarrow \infty$.

The interpretation of Sachs form factors as Fourier transforms of charge and magnetization densities in the nucleon requires to introduce localized nucleon states in the position space $|\vec{x}\rangle$ as opposed to the plane-wave states used above $|p\rangle$,

$|\vec{x}\rangle=\sum_{\vec{p}} \frac{\mathrm{e}^{i \vec{p} \cdot \vec{x}}}{\sqrt{V}} \Psi(\vec{p})|\vec{p}\rangle, \quad$ with $\quad \sum_{\vec{p}}|\Psi(\vec{p})|^{2}=1$

Here a very broad wave packet $\Psi(\vec{p}) \approx$ const is assumed in the momentum space. Then the charge density $\rho(\vec{x})$ of the nucleon, localized at $\vec{x}=0$, is

$$
\left\langle\vec{x}=0\left|j_{0}(\vec{x})\right| \vec{x}=0\right\rangle \equiv \tilde{\varphi}_{2}^{*} \tilde{\varphi}_{1} \rho(\vec{x})=\tilde{\varphi}_{2}^{*} \tilde{\varphi}_{1} \int \frac{d^{3} \vec{\Delta}}{(2 \pi)^{3}} \mathrm{e}^{-i \vec{\Delta} \cdot \vec{x}} G_{E}\left(-\vec{\Delta}^{2}\right),
$$

and similar for the magnetic form factor. The famous Hofstadter's experiments established that the proton is not a point-like particle $\rho_{\text {point }}(\vec{x})=\delta^{3}(\vec{x})$ which would have $G_{E}^{\text {point }}=$ const, but rather $G_{E}\left(-\vec{\Delta}^{2}\right) \approx\left(1+\vec{\Delta}^{2} r_{N}^{2} / 6\right)^{-2}$ with the mean square radius $r_{N} \approx 0.7 \mathrm{fm}$.

The Breit frame is not particularly instructive for an interpretation of high-energy scattering. Here an infinite momentum frame (IMF) is more useful, see discussion below. In this frame, obtained by a $z$-boost, the nucleon momentum is $p_{z}=\left(p_{1}+p_{2}\right)_{z} \rightarrow \infty$. In the IMF one builds a nucleon state localized in the transverse plane at $\boldsymbol{b}_{\perp}=(x, y)$

$\left|p_{z}, \boldsymbol{b}_{\perp}\right\rangle=\sum_{\boldsymbol{p}_{\perp}} \frac{\mathrm{e}^{i \boldsymbol{p}_{\perp} \cdot \boldsymbol{b}_{\perp}}}{\sqrt{V_{\perp}}} \Psi\left(\boldsymbol{p}_{\perp}\right)\left|p_{z}, \boldsymbol{p}_{\perp}\right\rangle$.

Then one finds that the transverse charge distribution of the nucleon wave packet, see Fig. Q, is given by the two-dimensional Fourier transform of form factors

$\left\langle p_{z}, \boldsymbol{b}_{\perp}=0\left|j_{+}\left(\boldsymbol{b}_{\perp}\right)\right| p_{z}, \boldsymbol{b}_{\perp}=0\right\rangle=h_{+} \int \frac{d^{2} \boldsymbol{\Delta}_{\perp}}{(2 \pi)^{2}} \mathrm{e}^{-i \boldsymbol{\Delta}_{\perp} \cdot \boldsymbol{b}_{\perp}} F_{1}\left(-\boldsymbol{\Delta}_{\perp}^{2}\right)+\ldots$

As previously one assumes a rather delocalized transverse momentum wave function $\sum_{\boldsymbol{p}_{\perp}} \Psi^{*}\left(\boldsymbol{p}_{\perp}+\boldsymbol{\Delta}_{\perp} / 2\right) \Psi\left(\boldsymbol{p}_{\perp}-\boldsymbol{\Delta}_{\perp} / 2\right) \approx 1$. Thus, we can interpret form factors as describing the transverse localization of partons in a fast moving nucleon, irrespective of their longitudinal momenta and independent on the resolution scale. 


\section{Parton densities}

The deeply inelastic lepton-nucleon scattering $\ell N \rightarrow \ell^{\prime} X$ probes, via the amplitude

$\mathcal{A}_{N X}=\frac{1}{Q^{2}} L_{\mu}(q)\left\langle p_{X}\left|j_{\mu}(0)\right| p\right\rangle$,

the nucleon with the resolution $\hbar / Q \approx(0.2 \mathrm{fm}) /(Q$ in $\mathrm{GeV})$, set by the photon virtuality $q^{2} \equiv-Q^{2}$. Recalling that the nucleon's size is $r_{N} \sim 1 \mathrm{fm}$, one concludes that for $Q^{2}$ of order of a few $\mathrm{GeV}$, the photon penetrates the nucleon interior and interacts with its constituents. The cross section of the deeply inelastic scattering is related, by the optical theorem, to the imaginary part of the forward Compton scattering amplitude

$d \sigma_{\text {DIS }}\left(x_{\mathrm{B}}, Q^{2}\right) \sim \sum_{X}\left|\mathcal{A}_{N X}\right|^{2} \delta^{4}\left(p+q-p_{X}\right) \sim \frac{1}{\pi} \Im \mathrm{m} i \int d^{4} z \mathrm{e}^{i q \cdot z}\left\langle p\left|T\left\{j_{\mu}^{\dagger}(z) j_{\mu}(0)\right\}\right| p\right\rangle$.

The very intuitive parton interpretation has its clear-cut meaning in the IMF. A typical interaction time of partons is inversely proportional to the energy deficit of a given fluctuation of a particle with the energy $E_{0}$ and three-momentum $\boldsymbol{p}_{0}=\left(\boldsymbol{p}_{\perp 0}, x_{0} p_{z}\right)$ into two partons with energies $E_{1,2}$ and three-momenta $\boldsymbol{p}_{1,2}=\left(\boldsymbol{p}_{\perp 1,2}, x_{1,2} p_{z}\right)$. It scales, for $p_{z} \rightarrow \infty$, as

$\Delta t \sim \frac{1}{\Delta E}=\frac{1}{E_{0}-E_{1}-E_{2}} \sim \frac{p_{z}}{\boldsymbol{p}_{\perp 0}^{2} / x_{0}-\boldsymbol{p}_{\perp 1}^{2} / x_{1}-\boldsymbol{p}_{\perp 2}^{2} / x_{2}} \rightarrow \infty$.

Therefore, one can treat partons as almost free in the IMF due to the time dilation. The virtual photon "sees" nucleon's constituents in a frozen state during the time of transiting the target which is, thus, describable by an instantaneous distribution of partons. Here again the analogy with X-ray crystallography is quite instructive: Recall that an X-ray, scattered off atoms, reveals crystal's structure since rapid oscillations of atoms in the lattice sites can be neglected. Atoms can be considered being at rest during the time $\mathrm{X}$-rays cross the crystal. The transverse distance probed by the virtual photon in a Lorentz contracted hadron, is of order $\delta z_{\perp} \sim 1 / Q$, see Fig. 2. One can conclude therefore that simultaneous scattering off an $n$-parton cascade is suppressed by an extra power of $\left(1 / Q^{2}\right)^{n-1}$. The leading contribution to $d \sigma_{\text {DIS }}$ is thus given by a handbag diagram, i.e., the photon-single-quark Compton amplitude. The character of relevant distances in the Compton amplitude (9) is a consequence of the Bjorken limit which implies large $Q^{2}$ (small distances) and energies $\nu \equiv p \cdot q$ (small times) at fixed $x_{\mathrm{B}} \equiv Q^{2} /(2 \nu)$. By going to the target rest frame one immediately finds that at large $Q^{2}$ the dominant contribution comes from the light-cone distances $z^{2} \approx \mathcal{O}\left(1 / Q^{2}\right)$ between the points of absorption and emission of the virtual photon in (9) because $z_{-} \sim 1 /\left(M x_{\mathrm{B}}\right), z_{+} \sim M x_{\mathrm{B}} / Q^{2}$.

Since the hard quark-photon subprocess occupies a very small space-time volume but the scales involved in the formation of the nucleon are much larger, hence, they are uncorrelated and will not interfere. The quantum mechanical incoherence of physics at different scales results into the factorization property of the cross section (9),

$d \sigma_{\text {DIS }}\left(x_{\mathrm{B}}, Q^{2}\right) \sim \sum_{q} e_{q}^{2} \int_{0}^{1} d x \delta\left(x-x_{\mathrm{B}}\right) f_{q}\left(x ; Q^{2}\right)$,

where $f_{q}$ is a parton distribution, - the density of probability to find partons of a given longitudinal momentum fraction $x$ of the parent nucleon with transverse resolution $1 / Q$, 

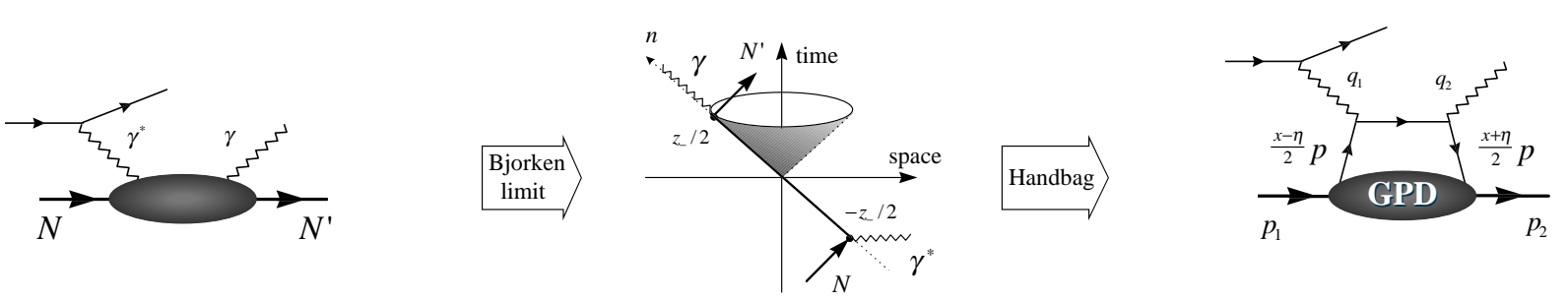

Figure 3. Light-cone dominance in deeply virtual Compton scatering.

$$
\left\langle p\left|\bar{q}(0) \gamma_{+} q\left(z_{-} n\right)\right| p\right\rangle=2 p_{+} \int_{0}^{1} d x\left\{f_{q}(x) \mathrm{e}^{-i x z_{-} p_{+}}-\bar{f}_{q}(x) \mathrm{e}^{i x z_{-} p_{+}}\right\} .
$$

No information on the transverse position of partons is accessible here, Fig. 2.

\section{Generalized parton distributions}

Both observables addressed in the previous two sections give only one-dimensional slices of the nucleon since only the magnitude of scattering amplitudes is accessed in the processes but its phase is lost. These orthogonal spaces are probed simultaneously in generalized parton distributions (GPDs), which arise in the description of deeply virtual Compton scattering (DVCS) $\ell N \rightarrow \ell^{\prime} \gamma^{*} N \rightarrow \ell^{\prime} N^{\prime} \gamma$ in the Bjorken limit, see Fig. 3. In the same spirit as in deeply inelastic scattering, the latter consists of sending $q^{2} \equiv\left(q_{1}+q_{2}\right)^{2} / 4 \rightarrow$ $-\infty$ to the deep Euclidean domain while keeping $\Delta^{2} \equiv\left(p_{2}-p_{1}\right)^{2} \ll-q^{2}$ small and $\xi \equiv-q^{2} / p \cdot q$ fixed, $p \equiv p_{1}+p_{2}$. By the reasoning along the same line as in the previous section one finds that the Compton amplitude factorizes into GPDs parametrizing the twist-two light-ray operator matrix element

$$
\left\langle p_{2}\left|\bar{q}\left(-z_{-} n\right) \gamma_{+} q\left(z_{-} n\right)\right| p_{1}\right\rangle=\int_{-1}^{1} d x \mathrm{e}^{-i x z_{-} p_{+}}\left\{h_{+} H_{q}\left(x, \eta, \Delta^{2}\right)+e_{+} E_{q}\left(x, \eta, \Delta^{2}\right)\right\}
$$

and a handbag coefficient function, so that one gets from Fig. 3

$$
\mathcal{A}_{\mathrm{DVCS}}=\varepsilon_{\mu}^{*}\left(q_{2}\right) L_{\nu}\left(q_{1}\right) \int d^{4} z \mathrm{e}^{i q \cdot z}\left\langle p_{2}\left|T\left\{j_{\mu}^{\dagger}(z / 2) j_{\nu}(-z / 2)\right\}\right| p_{1}\right\rangle \sim \sum_{q} e_{q}^{2} \int_{-1}^{1} d x \frac{F_{q}\left(x, \eta, \Delta^{2}\right)}{\xi-x-i 0},
$$

where $F_{q}=H_{q}, E_{q}$ and the contribution from a crossed diagram is omitted. GPDs depend on the $s$-channel momentum fraction $x$, measured with respect to the momentum $p$, and $t$-channel fraction $\eta \equiv q \cdot \Delta / q \cdot p$, which is the longitudinal component of the momentum transfer $\Delta \approx \eta p+\Delta_{\perp}$, as well as its square $\Delta^{2} \approx-\left(\Delta_{\perp}^{2}+4 M_{N}^{2} \eta^{2}\right) /\left(1-\eta^{2}\right)$. Due to the reality of the final state photon $\eta \approx-\xi$. A geometric picture underlying DVCS is as follows, see Fig. 4. The electric field of lepton's virtual fluctuation $\ell \rightarrow \ell^{\prime} \gamma^{*}$ accelerates a quark localized in the transverse area $\left(\delta z_{\perp}\right)^{2} \sim 1 / Q^{2}$ at the impact parameter $\boldsymbol{b}_{\perp}$ and carrying a certain momentum fraction of the parent nucleon. The accelerated parton tends to emit the energy via electromagnetic radiation and fall back into the nucleon, see Fig. 4. The incoming-outgoing nucleon system is localized at the center of coordinates $\boldsymbol{b}_{\perp}=0$, however, due to non-zero longitudinal momentum exchange in the $t$-channel the individual transverse localizations of incoming and outgoing nucleons are shifted in the transverse plane by amounts' $\Delta \boldsymbol{b}_{\perp} \sim \eta(1+\eta) \boldsymbol{b}_{\perp}$ and $\Delta \boldsymbol{b}_{\perp}^{\prime} \sim \eta(1-\eta) \boldsymbol{b}_{\perp}$, respectively [2].

\footnotetext{
${ }^{1}$ Note the difference in the definition of our impact parameter space GPDs as compared to Ref. [2]. In our frame $\boldsymbol{p}_{\perp 2}=-\boldsymbol{p}_{\perp 1}=\boldsymbol{\Delta}_{\perp} / 2$. We define the Fourier transform with respect to $\boldsymbol{\Delta}_{\perp}$ which has its
} 


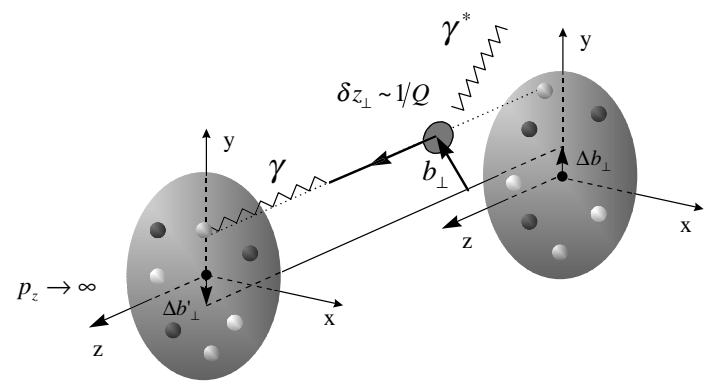

Figure 4. Geometric picture of deeply virtual Compton scattering.

Generally, GPDs are not probabilities rather they are the interference of amplitudes of removing a parton with one momentum and inserting it back with another. In the limit $\Delta=0$ they reduce to inclusive parton densities and acquire the probabilistic interpretation. This is exhibited in a most straightforward way in the light-cone formalism [3], where one easily identifies the regions $-1<x<-\eta$ and $\eta<x<1$ with parton densities while $-\eta<x<\eta$ with distribution amplitudes. This latter domain precludes the density interpretation for $\eta \neq 0$.

The first moment of GPDs turns into form factors (11). The second moment of Eq. (13) gives form factors of the quark energy-momentum tensor, $\Theta_{\mu \nu}^{q}=\bar{q} \gamma_{\{\mu} D_{\nu\}} q$. Since gravity couples to matter via $\Theta_{\mu \nu}=\frac{\delta}{\delta g_{\mu \nu}} \int d^{4} x \sqrt{-\operatorname{det} g_{\mu \nu}(x)} \mathcal{L}(x)$, these form factors are the ones of the nucleon scattering in a weak gravitational field [4]

$\left\langle p_{2}\left|\Theta_{\mu \nu}\right| p_{1}\right\rangle=A\left(\Delta^{2}\right) h_{\{\mu} p_{\nu\}}+B\left(\Delta^{2}\right) e_{\{\mu} p_{\nu\}}+C\left(\Delta^{2}\right) \Delta_{\mu} \Delta_{\nu}$.

Analogously to the previously discussed electromagnetic case, the combination $A\left(\Delta^{2}\right)+$ $\Delta^{2} /\left(4 M_{N}^{2}\right) B\left(\Delta^{2}\right)$ arising in the $\Theta_{00}$ component measures the mass distribution inside the nucleon [5]. It is different from the charge distribution due to presence of neutral constituents inside hadrons not accounted in electromegnetic form factors. The gravitomagnetic form factor $A\left(\Delta^{2}\right)+B\left(\Delta^{2}\right)$ at zero recoil encodes information on the parton angular momentum [4] $\vec{J}=\frac{1}{V} \int d^{3} \vec{x}[\vec{x} \times \vec{\Theta}](x)$ expressed in terms of the momentum flow operator $\Theta_{0 i} \equiv \Theta_{i}$ in the nucleon and gives its distribution when Fourier transformed to the coordinate space. These form factor are accessible once GPDs are measured:

$\int_{-1}^{1} d x x H\left(x, \eta, \Delta^{2}\right)=A\left(\Delta^{2}\right)+\eta^{2} C\left(\Delta^{2}\right), \quad \int_{-1}^{1} d x x E\left(x, \eta, \Delta^{2}\right)=B\left(\Delta^{2}\right)-\eta^{2} C\left(\Delta^{2}\right) .(15$

GPDs regain a probabilistic interpretation once one sets $\eta=0$ but $\boldsymbol{\Delta}_{\perp} \neq 0$ [6]:7]. When Fourier transformed to the impact parameter space they give a very intuitive picture of measuring partons of momentum fraction $x$ at the impact parameter $\boldsymbol{b}_{\perp}$ with the resolution of order $1 / Q$ set by the photon virtuality in the localized nucleon state (6),

$f\left(x, \boldsymbol{b}_{\perp}\right)=\int \frac{d^{2} \boldsymbol{\Delta}_{\perp}}{(2 \pi)^{2}} \mathrm{e}^{-i \boldsymbol{\Delta}_{\perp} \cdot \boldsymbol{b}_{\perp}} H\left(x, 0,-\boldsymbol{\Delta}_{\perp}^{2}\right)$.

To visualize it, see Fig. 2, one can stick to the Regge-motivated ansatz $H\left(x, 0,-\boldsymbol{\Delta}_{\perp}^{2}\right) \sim$ $x^{-\alpha_{R}\left(-\Delta_{\perp}^{2}\right)}(1-x)^{3}$ with a linear trajectory $\alpha_{R}\left(-\Delta_{\perp}^{2}\right)=\alpha_{R}(0)-\alpha_{R}^{\prime} \Delta_{\perp}^{2}$ where $\alpha_{R}(0) \approx 0.5$ and $\alpha^{\prime} \approx 1 \mathrm{GeV}^{2}$.

advantages that $\boldsymbol{b}_{\perp}$ does not depend implicitly on $\eta$. 

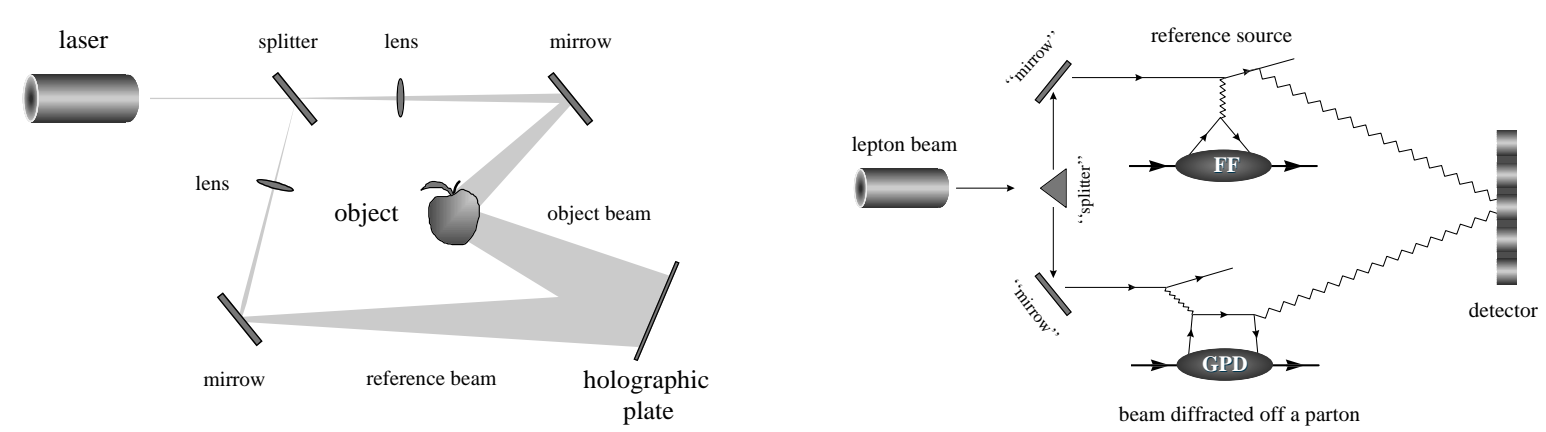

Figure 5. Left: Conventional setup for taking the holographic picture. Right: Nucleon hologram with leptoproduction of a photon: interference of the Bethe-Heitler (reference) and DVCS (sample) amplitudes.

\section{Hard leptoproduction of real photon and lepton pair}

The light-cone dominance in DVCS is a consequence of the external kinematical conditions on the process in the same way as in deeply inelastic scattering. Therefore, one can expect precocious scaling starting as early as at $-q^{2} \sim 1 \mathrm{GeV}^{2}$. It is not the case for hard exclusive meson production, giving access to GPDs as well, where it is the dynamical behavior of the short-distance parton amplitude confined to a small transverse volume near the light cone that drives the perturbative approach to the process. Here the reliability of perturbative QCD predictions is postponed to larger momentum transfer.

Although GPDs carry information on both longitudinal and transverse degrees of freedom, their three-dimensional experimental exploration requires a complete determination of the DVCS amplitude, i.e., its magnitude and phase. One way to measure the phase at a given spot is known as holography, for visible light. This technology allows to make threedimensional photographs of objects, see Fig. 5: The laser beam splits into two rays. One of them serves as a reference source and the other reflects from the object's surface. The reflected beam, which was in phase with the reference beam before hitting the "target", interferes with the reference beam and forms fringes on the plate with varying intensity depending on the phase difference of both. (Unfortunately, the same method cannot be used for X-ray holography of crystals and scattering experiments due to the absence of practical "splitters".) For the exclusive leptoproduction of a photon, however, there are two contributions to the amplitude: the DVCS one $\mathcal{A}_{\mathrm{DVCS}}$, we are interested in, and $\mathcal{A}_{\mathrm{BH}}$ from the 'contaminating' Bethe-Heitler (BH) process, in which the real photon spills off the scattered lepton rather than the quark, see Fig. 5. The BH amplitude is completely known since the only long-distance input turns out to be nucleon form factors measured elsewhere. The relative phase of the amplitudes can be measured by the interference of DVCS and BH amplitudes in the cross section $d \sigma_{\ell N \rightarrow \ell^{\prime} N^{\prime} \gamma} \sim\left|\mathcal{A}_{\mathrm{DVCS}}+\mathcal{A}_{\mathrm{BH}}\right|^{2}$ and, thus, the nucleon hologram can be taken. The most straightforward extraction of the interference term is achieved by making use of the opposite lepton charge conjugation properties of DVCS and BH amplitudes. The former is odd while the latter is even under change of the lepton charge. The unpolarized beam charge asymmetry gives

$$
d \sigma_{\ell N \rightarrow \ell^{\prime} N^{\prime} \gamma}\left(+e_{\ell}\right)-d \sigma_{\ell N \rightarrow \ell^{\prime} N^{\prime} \gamma}\left(-e_{\ell}\right)=\left(\mathcal{A}_{\mathrm{DVCS}}+\mathcal{A}_{\mathrm{DVCS}}^{*}\right) \mathcal{A}_{\mathrm{BH}} \sim \Re \mathrm{e} \int \frac{F_{q}\left(x, \xi, \Delta^{2}\right)}{\xi-x-i 0} \cos \phi_{\gamma}^{\prime}
$$



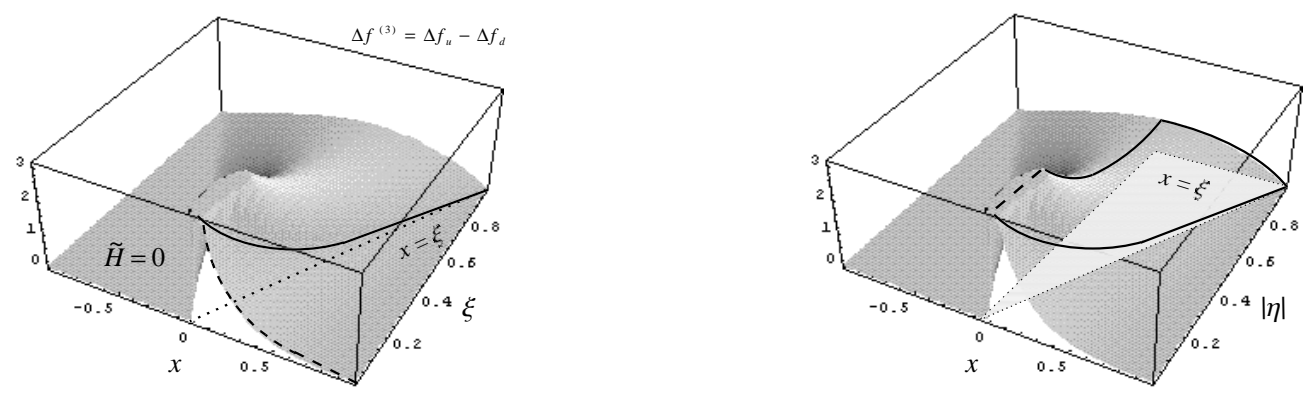

Figure 6. What is extractable from DVCS (left) and DVCS lepton pair production (right).

and measures the real part of the DVCS amplitude modulated by the harmonics of the azimuthal angle between the lepton and photon scattering planes $\phi_{\gamma}^{\prime}$ [8.9]. If on top of the charge asymmetry one further forms either beam or target polarization differences, this procedure would allow to cleanly extract the imaginary part of the DVCS amplitude where GPDs enter in diverse combinations. These rather involved measurements have not yet been done. Luckily, since the ratio of BH to DVCS amplitude scales like $\left[\Delta^{2} / q_{1}^{2}(1-\right.$ $y)]^{1 / 2} / y$, for large $y$ or small $-\Delta^{2}$, it is safe to neglect $\left|\mathcal{A}_{\mathrm{DVCS}}\right|^{2}$ as compared to other terms. Thus, in such kinematical settings one has access to the interference in single spin asymmetries,

$d \sigma_{\ell N \rightarrow \ell^{\prime} N^{\prime} \gamma}\left(+\lambda_{\ell}\right)-d \sigma_{\ell N \rightarrow \ell^{\prime} N^{\prime} \gamma}\left(-\lambda_{\ell}\right) \approx\left(\mathcal{A}_{\mathrm{DVCS}}-\mathcal{A}_{\mathrm{DVCS}}^{*}\right) \mathcal{A}_{\mathrm{BH}} \sim \Im \mathrm{m} \int \frac{F_{q}\left(x, \xi, \Delta^{2}\right)}{\xi-x-i 0} \sin \phi_{\gamma}^{\prime}$

which measure GPDs directly on the line $x=\xi$ as shown in Fig. 6. Experimental measurements of these asymmetries were done by HERMES [11,13] and CLAS [12] collabarations. The comparison to current GPD models is demonstrated in Fig. 0 .

In order to go off the diagonal $x=\xi$ one has to relax the reality constraint on the outgoing $\gamma$-quantum, i.e., it has to be virtual and fragment into a lepton pair $\bar{L} L$ with invariant mass $q_{2}^{2}>0$. Thus, one has to study the process $\ell N \rightarrow \ell^{\prime} \bar{L} L N^{\prime}$. In these circumstances, the skewedness parameter $\eta$ independently varies for fixed Bjorken variable since $\xi \approx-\eta\left(\left|q_{1}^{2}\right|-q_{2}^{2}\right) /\left(\left|q_{1}^{2}\right|+q_{2}^{2}\right)$, and one is able to scan the three-dimensional shape of GPDs, see Fig. 6. Unfortunately, the cross section for DVCS lepton pair production is suppressed by $\alpha_{\mathrm{em}}^{2}$ as compared to DVCS and also suffers from resonance backgrounds, see, e.g., [14].

Finally, perturbative next-to-leading (NLO) and higher-twist effects are shortly discussed. Estimates of the former are, in general, model dependent. NLO contributions to the hard-scattering amplitude [15] of a given quark species are rather moderate, i.e., of the relative size of $20 \%$, however, the net result in the DVCS amplitude can be accidentally large [8,16]. This can be caused by a partial cancellation that occurs in tree amplitudes. Evolution effects [17] in the flavor non-singlet sector are rather small. In the case of gluonic GPD models we observed rather large NLO corrections to the DVCS amplitude for the naive scale setting $\mu_{F}^{2}=-q_{1}^{2}[8]$. For such models one also has rather strong evolution effects, which severely affect LO analysis. However, one can tune the factorization scale $\mu_{F}$ so that to get rid of these effects. The renormalon-motivated twist-four [18] and target mass corrections [19] await their quantitative exploration. 

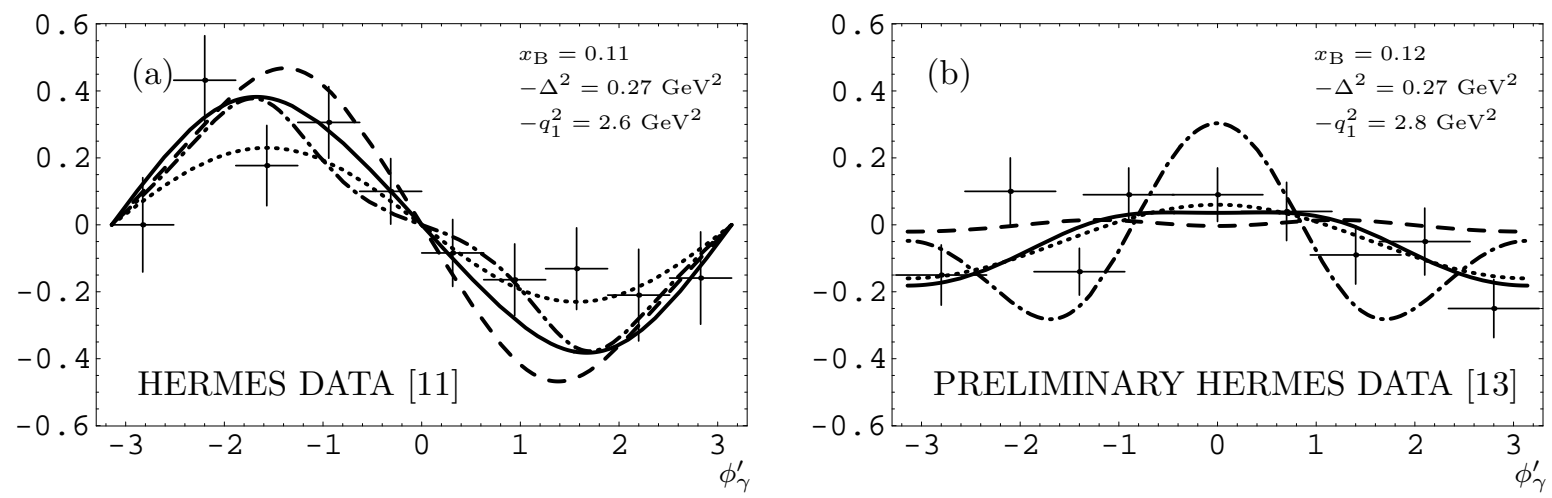

Figure 7. Beam spin asymmetry (a) in $e^{+} p \rightarrow e^{+} p \gamma$ and unpolarized charge asymmetry (b) from HERMES with $E=27.6 \mathrm{GeV}$ are predicted making use of the complete twistthree analysis for input GPDs from Ref. [8]: model A without the D-term (solid) and $\mathrm{C}$ with the D-term (dashed) in the Wandzura-Wilczek approximation [10] as well as the model B with the D-term (dash-dotted) and included quark-gluon correlations. The dotted lines on the left and right panels show $0.23 \sin \phi_{\gamma}^{\prime}$ and $-0.05+0.11 \cos \phi_{\gamma}^{\prime}$ HERMES fits, respectively. Note that a toy model for quark-gluon correlations while only slightly changing the beam asymmetry, however, strongly alter the charge asymmetry.

\section{REFERENCES}

1. D. Müller et al., Fortschr. Phys. 42 (1994) 101; X. Ji, Phys. Rev. D 55 (1997) 7114; A.V. Radyushkin, Phys. Rev. D 56 (1997) 5524.

2. M. Diehl, hep-ph/0205208;

3. M. Diehl et al., Nucl. Phys. B 596 (2001) 33; S.J. Brodsky, M. Diehl, D.S. Hwang, Nucl. Phys. B 596 (2001) 99.

4. X. Ji, Phys. Rev. Lett. 78 (1997) 610.

5. A.V. Belitsky, X. Ji, Phys. Lett. B 538 (2002) 289.

6. M. Burkardt, Phys. Rev. D 62 (2000) 071503; hep-ph/0105324; hep-ph/0207047.

7. J.P. Ralston, B. Pire, hep-ph/0110075.

8. A.V. Belitsky, D. Müller, A. Kirchner, Nucl. Phys. B 629 (2002) 323.

9. M. Diehl et al., Phys. Lett. B 411 (1997) 193; A.V. Belitsky et al., Nucl. Phys. B 593 (2001) 289.

10. A.V. Belitsky, D. Müller, Nucl. Phys. B 589 (2000) 611; N. Kivel et al., Phys. Lett. B 497 (2001) 73; A.V. Radyushkin, C. Weiss, Phys. Rev. D 63 (2001) 114012.

11. A. Airapetian et al. (HERMES Coll.), Phys. Rev. Lett. 87 (2001) 182001.

12. S. Stepanyan et al. (CLAS Coll.), Phys. Rev. Lett. 87 (2001) 182002.

13. F. Ellinghaus, these proceedings; hep-ex/0207029.

14. E.R. Berger, M. Diehl, B. Pire, Eur. Phys. J. C 23 (2002) 675.

15. A.V. Belitsky, D. Müller, Phys. Lett. B 417 (1997) 129; L. Mankiewicz et al., Phys. Lett. B 425 (1998) 186; X. Ji, J. Osborne, Phys. Rev. D 58 (1998) 094018.

16. A. Freund, M. McDermott, Phys. Rev. D 65 (2002) 074008.

17. D. Müller, Phys. Rev. D 49 (1994) 2525; A.V. Belitsky, D. Müller, Nucl. Phys. B 537 (1999) 397; A.V. Belitsky, A. Freund, D. Müller, Nucl. Phys. B 574 (2000) 347.

18. A.V. Belitsky, A. Schäfer, Nucl. Phys. B 527 (1998) 235.

19. A.V. Belitsky, D. Müller, Phys. Lett. B 507 (2001) 173. 